\title{
Cuidado de enfermagem em gestantes com câncer de mama: revisão integrativa
}

\author{
Nursing care in pregnant women with breast cancer: integrative review \\ Atención a las enfermedades de las embarazadas con cáncer de mama: una revisión integradora
}

Recebido: 30/11/2021 | Revisado: 06/12/2021 | Aceito: 07/12/2021 | Publicado: 14/12/2021

Luciana Soares da Silva

ORCID: https://orcid.org/0000-0001-7962-4198 Centro Universitário da Amazônia, Brasil E-mail:lucianasoares116@gmail.com

Pablo Miranda Freitas

ORCID: https://orcid.org/0000-0001-8990-330X Centro Universitário da Amazônia, Brasil E-mail:pablodemiranda2017@gmail.com

Adria Leitão Maia

ORCID: https://orcid.org/0000-0002-4173-1374 Centro Universitário da Amazônia, Brasil E-mail: adria-maia@hotmail.com

\begin{abstract}
Resumo
Objetivo: Verificar quais cuidados precisam ser adotadospela equipe de Enfermagem às gestantes com diagnóstico de câncer de mama durante o período gestacional, analisar as estratégias utilizadas pelos enfermeiros em vista de assistência holística considerando suas vulnerabilidades e analisar o papel da Enfermagem na atuação e tratamento da portadora de neoplasia mamária no período gravídico. Método: De cunho bibliográfico, com levantamento de dados nas bases, Biblioteca Virtual em Saúde (BVS), Biblioteca Científica Eletrônica Online (SciELO), Literatura Latino-Americano e do Caribe (LILACS) e PUBMED; os descritores em saúde (DeCS) foram: Neoplasias da mama, Gravidez, Manutenção da Gravidez, Planejamento de enfermagem ao paciente e Enfermagem oncológica. Resultado: A neoplasiamamária é o segundo câncer mais presente no mundo entre a população feminina. No Brasil, os índices de mortalidade por neoplasia mamária têm tomado uma grande proporção. O tratamento é multifatorial e individual, o papel do enfermeiro se constrói na condução de situações relacionadas ao câncer na gestação sendo essencial para a paciente, promovendo informações acerca do autocuidado, oferecendo apoio emocional, proporcionando alívio da dor, incentivando e encorajamento. Conclusão: Ressalta-se que a Enfermagem está à frente do cuidado à mulher em todos os níveis de atenção, motivo pelo qual esses profissionais precisam estar qualificados e atentos, seja ela qual a situação. A relevância da prevenção ou a detecção precoce da neoplasia mamária no período gestacional, traz consigo fatores positivos para o prognóstico da doença.
\end{abstract}

Palavras-chave: Neoplasias da mama; Gravidez; Manutenção da gravidez; Planejamento de assistência ao paciente; Enfermagem oncológica.

\begin{abstract}
Objective: To verify what care needs to be taken by the Nursing team to pregnant women diagnosed with breast cancer during pregnancy, to analyze the strategies used by nurses in view of holistic care considering their vulnerabilities and to analyze the role of Nursing in the performance and treatment of with breast cancer during pregnancy. Method: Bibliographic in nature, with data collection in the databases, Virtual Health Library (VHL), Electronic Scientific Library Online (SciELO), Latin American and Caribbean Literature (LILACS) and PUBMED; the descriptors in health (DeCS) were: Breast neoplasms, Pregnancy, Pregnancy Maintenance, Patient nursing planning and Oncology nursing. Result: Breast cancer is the second most prevalent cancer in the world among the female population. In Brazil, mortality rates for breast cancer have taken a large proportion. The treatment is multifactorial and individual, the role of the nurse is built in conducting situations related to cancer during pregnancy, being essential for the patient, promoting information about self-care, offering emotional support, providing pain relief, encouraging and encouraging. Conclusion: It is noteworthy that Nursing is at the forefront of care for women at all levels of care, which is why these professionals need to be qualified and attentive, whatever the situation. The relevance of prevention or early detection of breast cancer during pregnancy brings with it positive factors for the prognosis of the disease.
\end{abstract}

Keywords: Breast neoplasms; Pregnancy; Pregnancy maintenance; Patient care planning; Oncology nursing.

\section{Resumen}

Objetivo: Verificar qué cuidados deben ser cuidados por el equipo de Enfermería a las gestantes diagnosticadas de cáncer de mama durante el embarazo, analizar las estrategias utilizadas por las enfermeras ante la atención integral considerando sus vulnerabilidades y analizar el rol de la Enfermería en el desempeño y tratamiento del cáncer de mama durante el 
embarazo. Método: De naturaleza bibliográfica, con recolección de datos en las bases de datos, Biblioteca Virtual en Salud (BVS), Biblioteca Científica Electrónica en Línea (SciELO), Literatura Latinoamericana y del Caribe (LILACS) y PUBMED; los descriptores en salud (DeCS) fueron: neoplasias mamarias, embarazo, mantenimiento del embarazo, planificación de enfermería del paciente y enfermería oncológica. Resultado: El cáncer de mama es el segundo cáncer más prevalente en el mundo entre la población femenina. En Brasil, las tasas de mortalidad por cáncer de mama han tomado una gran proporción. El tratamiento es multifactorial e individual, el rol de la enfermera se construye en la conducción de situaciones relacionadas con el cáncer durante el embarazo, siendo fundamental para la paciente, promoviendo información sobre el autocuidado, ofreciendo apoyo emocional, brindando alivio del dolor, alentando y alentando. Conclusión: Es de destacar que la Enfermería está a la vanguardia de la atención a la mujer en todos los niveles de atención, por lo que estos profesionales necesitan estar calificados y atentos, sea cual sea la situación. La relevancia de la prevención o detección precoz del cáncer de mama durante el embarazo trae consigo factores positivos para el pronóstico de la enfermedad.

Palabras clave: Neoplasias de mama; Embarazo; Mantenimiento del embarazo; Planificación de atención al paciente; Enfermería oncológica.

\section{Introdução}

Câncer (CA) é uma patologia que desenvolve o crescimento anormal das células. Na atualidade, refere-se a um problema de saúde pública com uma grande relevância na porcentagem de óbitos registrados (Braga et al, 2018).

O câncer equivale a $21 \%$ dos números de óbitos no mundo, essa estimativa ocorre principalmente em países pouco desenvolvidos. Destaca-se que o câncer de mama é a segunda causa de neoplasia na gravidez, levando em consideração esses números, o câncer de mama ocorre comumente nesta fase (Prado et al, 2020).

A descoberta e a vivência do câncer de mama associado a gestação se revela em um momento delicado e de grandes desafios. Tal situação evolui para um momento com alta fragilidade associados a uma vulnerabilidade emocional para a gestante e seus familiares, com várias consequências, que pode ocorrer por falta de conhecimentos e informações sobre como o tratamento pode afetar diretamente a saúde fetal (Braga et al, 2018).

Dessa forma Lima e Pontes (2019), complementam que o índice de câncer na gravidez pode levar a um aumento consequente na transformação da sociedade econômica e social, com os novos hábitos modernos de vida das mulheres, isto é, com o menor número de paridade e com o adiamento da primeira gestação associados à propensão genética e hábitos da mulher moderna.

Ainda de acordo com Lima e Pontes (2019), é de extrema importância que os profissionais Enfermeiros envolvidos nesse processo do período gestacional e câncer de mama, desenvolvam uma assistência especializada com decisões voltadas para os princípios éticos, legais e científicos, tudo direcionado para a situação atual e individual, estando dentre as atribuições do profissional Enfermeiro as práticas educativas para a promoção, prevenção e recuperação da saúde mãe-filho.

O impasse dos profissionais de saúde sobre uma assistência voltada para a gestante com câncer de mama, é não possuir experiências e treinamentos para desenvolver um cuidado especializado, pois a literatura ainda é muito escassa quando se trata de abordar temáticas que ligam esses dois assuntos, com isso o profissional também fica vulnerável diante do diagnóstico, de não saber como lidar com essa fase da gestação e o CA mamário, consequentemente não prestando uma assistência adequada e especializada para os envolvidos, mãe-feto e familiares (Cipriano \& Oliveira, 2016).

Diante do exposto, mesmo com os avanços e diversas alternativas para o cuidado puerperal, os Enfermeiros se destacam como os profissionais que orientam e ofertam osprimeiros suportes emocionais às mulheres e seus familiares. Conhecer a atuação dos profissionais Enfermeiros frente a gestantes portadoras do câncer de mama é de suma importância para a comunidade em geral. A Enfermagem ressurge como a primeira assistência para a grávida, que necessita de informações, tratando problemas emocionais, embate interior com o corpo físico da mulher, e com a incerteza de uma possível perda do feto. Por isso, percebe-se a necessidade de uma assistência de Enfermagem de qualidade envolvendo a gestante e seus familiares, com a tentativa de melhorar a expectativa de vida das grávidas com neoplasia mamária (Lima \& Pontes, 2019).

O presente estudo objetiva avaliar o perfil das gestantes mais acometidas pelo câncer de mama e sua grande importância 
para uma prevenção de CA mamário no período gestacional.

\section{Materiais e Métodos}

Nessa pesquisa, realizamos um estudo de revisão integrativa da literatura de caráter descritivo com abordagem bibliográfica do tipo qualitativa e exploratório que busca investigar o cuidado de enfermagem em gestantes com neoplasia mamária.

De acordo com Soares et al (2018), a pesquisa bibliográfica expõe sinais que visam buscar por referencial bibliográfico em amplas fontes. A pesquisa de bibliografia é essencial para a execução de estudos monográficos, ressaltando que o levantamento de estudos bibliográficos é o pilar do estudo exploratório, contendo anotações, registros, notas de aulas e a visão crítica que seja associado ao tema de interesse, de forma que a contribuição seja memorável para os registros.

$\mathrm{O}$ artigo de revisão pela pesquisa bibliográfica, tem como características a busca de referenciais em diversas fontes, para que os autores expressem sua linha de pensamento sobre o mesmo assunto, com ideias e visão diferente (Soares et al, 2018).

Acerca do estudo abordado referente ao cuidado de enfermagem em gestantes com câncer de mama, foram selecionados artigos publicados em revistas científicas, e posteriormente realizada a seleção e classificação dos mesmos. Para a realização de coleta de dados foi realizado um levantamento de informações relacionados ao objeto de estudo, através de artigos em bases de dados eletrônicos: Biblioteca Virtual em Saúde (BVS), Bireme, Google Acadêmico, Biblioteca Científica Eletrônica Online (SciELO), Literatura Latino-Americano e do Caribe (LILACS) e PUBMED.

O levantamento bibliográfico para a construção desse projeto foi realizado entre os meses de março a abril de 2021. Por ser um estudo de revisão bibliográfica, os dados são de fontes literárias, que contenham relação com o tema escolhido e que sejam dos anos de 2016 a 2021.

Os descritores utilizados para as buscas, foram: "Neoplasias da mama, gravidez, manutenção da gravidez, planejamento de assistência ao paciente e enfermagem oncológica". A construção deste trabalho acadêmico envolve pesquisas que buscam fundamentar, e amparar a produção em determinada área de conhecimento.

\section{Resultados e Discussões}

Com intuito de elucidar os resultados, foram retratados no Quadro 1 os 10 artigos selecionados. Os dados relacionados são: autores, título do artigo, ano, objetivos e resultados.

Quadro 1: Síntese de Artigos selecionados para composição de Resultados e Discussões sobre o Câncer de Mama no período gestacional.

\begin{tabular}{|c|c|c|c|c|}
\hline AUTORES & TÍTULO & ANO & OBJETIVO & RESULTADOS \\
\hline $\begin{array}{l}\text { MAYAAN, } \\
\text { Sanjaya; et } \\
\text { al }\end{array}$ & $\begin{array}{l}\text { Câncer } \\
\text { Gestacional - } \\
\text { Importância do } \\
\text { conhecimen to e } \\
\text { aprimoramento da } \\
\text { equipe } \\
\text { enfermagem }\end{array}$ & 2019 & $\begin{array}{l}\text { Proporcionar uma melhor } \\
\text { realização da sistematização da } \\
\text { Assistência de Enfermagem, como } \\
\text { condutas, diagnósticos de } \\
\text { enfermagem, prescrição, } \\
\text { implementação e avaliação, para } \\
\text { melhor identificação de gestantes } \\
\text { com câncer. }\end{array}$ & $\begin{array}{l}\text { Os autores concluíram o trabalho, afirmando que o papel da } \\
\text { enfermagem, torna-se essencial nas condutas em pacientes gestantes } \\
\text { com câncer, onde a atenção deverá ser redobrada e esse profissional } \\
\text { necessita de um conhecimento cientifico contínuo e holístico sobre } \\
\text { os efeitos colaterais das medicações administradas, dos } \\
\text { quimioterápicos, dos procedimentos terapêuticos, apoio emocional } \\
\text { e esclarecimento sobre a doença e suas possíveis consequências, } \\
\text { incentivando e encorajando a paciente enfrentá-la. }\end{array}$ \\
\hline $\begin{array}{l}\text { PRADO, } \\
\text { Natalia; } \\
\text { et al }\end{array}$ & $\begin{array}{lr}\text { Gestante } & \text { com } \\
\text { diagnóstico } & \text { de } \\
\text { câncer de mama: } \\
\text { prevenção, } \\
\text { diagnóstico } \\
\text { assistência }\end{array}$ & 2020 & $\begin{array}{l}\text { O objetivo do texto é nos mostrar } \\
\text { através da literatura sobre a } \\
\text { prevenção, diagnóstico e } \\
\text { prognóstico de câncer de mama } \\
\text { durante a gestação. E também } \\
\text { conhecer a capacidade da equipe } \\
\text { de saúde/enfermagem na } \\
\text { assistência da mulher grávida com } \\
\text { câncer de mama. Retratar sobre as } \\
\text { fragilidades da grávida com } \\
\text { câncer de mama. Constatar as }\end{array}$ & $\begin{array}{l}\text { As análises indicam que uma das maiores interferências que leva a } \\
\text { mulher a não realização da mamografia é a falta de informação e } \\
\text { orientação dos profissionais da saúde, deixando-as envergonhadas e } \\
\text { desconfortáveis, ainda mais quando o enfermeiro é jovem e do sexo } \\
\text { masculino, causando insegurança devida à falta de informação. } \\
\text { Levando essa paciente criar uma barreira sobre a doença e descobrir } \\
\text { tardiamente o diagnóstico de câncer, sendo uma doença } \\
\text { degenerativa agravando de forma rápida. Outro fator é a idade da } \\
\text { paciente, pois a partir de } 50 \text { anos o índice de câncer aumenta } \\
\text { disparadamente. }\end{array}$ \\
\hline
\end{tabular}




\begin{tabular}{|c|c|c|c|c|}
\hline & & & $\begin{array}{l}\text { causas sugestionadas e sugeridas } \\
\text { no tratamento da grávida com } \\
\text { câncer. }\end{array}$ & \\
\hline $\begin{array}{l}\text { RODRIGU } \\
\text { ES, } \\
\text { C. Millena; } \\
\text { et al }\end{array}$ & $\begin{array}{lr}\text { Repercussão } & \text { do } \\
\text { tratamento das } \\
\text { neoplasias durante } \\
\text { a gestação }\end{array}$ & 2016 & $\begin{array}{l}\text { O questionamento que direcionou } \\
\text { esta pesquisa foi: Quais os } \\
\text { resultados reproduzidos do câncer } \\
\text { diagnosticado no período da } \\
\text { gravidez? A partir disso, essa } \\
\text { busca tem como intuito retratar, } \\
\text { por meio de uma retificação } \\
\text { integrativa, os tipos mais comuns } \\
\text { de canceres descobertos no } \\
\text { período da gravidez, assim como, } \\
\text { as principais formas de tratamento } \\
\text { do câncer em grávidas. }\end{array}$ & $\begin{array}{l}\text { De acordo com o artigo é necessário que o profissional enfermeiro } \\
\text { esteja capacitado e qualificado para executar suas atribuições } \\
\text { relacionadas ao câncer de mama durante a gestação, enfatizando o } \\
\text { exame clínico das mamas e o citológico que é um exame feito em } \\
\text { todas as gestantes. Vale ressaltar que, essa propedêutica é } \\
\text { competência do profissional de enfermagem, e consiste em } \\
\text { diagnosticar e detectar qualquer alteração ou complicação } \\
\text { direcionada as mamas durante o período gestacional, para assim } \\
\text { encaminhar a um serviço especializado para que breve comece o } \\
\text { tratamento evitando perdas de vidas. Foi observado que se torna } \\
\text { necessário uma capacitação contínua para o profissional enfermeiro } \\
\text { que realiza o pré-natal. São muitas as instigações enfrentadas, a } \\
\text { complexidade de realizar o diagnóstico, as precauções a serem } \\
\text { tomadas, as resoluções e os riscos em conservar a gravidez, a } \\
\text { continuação do tratamento. As lesões de baixo grau, em sua } \\
\text { maioria, voltam no decorrer do pós-parto com taxa de regressão } \\
\text { entre } 64 \text { e } 80 \% \text {, sendo que sua ocorrência de progressão para a lesão } \\
\text { de alto grau é de } 6 \% \text {. Então esse profissional precisa estar sempre } \\
\text { atualizado acerca do câncer, onde sua atribuição é enfatizar os } \\
\text { diversos exames necessários de maneira prática e minuciosa. }\end{array}$ \\
\hline $\begin{array}{l}\text { LIMA, } \\
\text { V. Cleia; } \\
\text { et al }\end{array}$ & $\begin{array}{l}\text { Enfrentamento da } \\
\text { mulher com } \\
\text { diagnóstico de } \\
\text { câncer no período } \\
\text { gestacional }\end{array}$ & 2019 & $\begin{array}{l}\text { A pesquisa tem como objetivo, } \\
\text { detectar na literatura cientifica } \\
\text { indicações do confronto da } \\
\text { gestante frente ao diagnóstico e } \\
\text { tratamento de câncer durante a } \\
\text { gravidez. }\end{array}$ & $\begin{array}{l}\text { Os autores concluíram o trabalho afirmando que é necessária uma } \\
\text { melhor preparação aos profissionais enfermeiros que estão na linha } \\
\text { de frente cuidando dessas gestantes, tendo comprometimento ético, } \\
\text { conhecimento científico para identificar possíveis fatores de risco e } \\
\text { acompanhar essa gestante no pré-natal durante todo o período } \\
\text { gestacional. }\end{array}$ \\
\hline $\begin{array}{l}\text { BRAGA, } \\
\text { F. Laís; } \\
\text { et al }\end{array}$ & $\begin{array}{l}\text { Orientações de } \\
\text { enfermagem para } \\
\text { enfrentamento do } \\
\text { câncer de mama na } \\
\text { gravidez }\end{array}$ & 2016 & $\begin{array}{l}\text { O objetivo desta investigação é } \\
\text { distinguir os sentimentos das } \\
\text { gestantes em tratamento de } \\
\text { neoplasias, demonstrar } \\
\text { importância do auxílio da } \\
\text { prescrição de enfermagem e } \\
\text { projetar uma melhor preparação } \\
\text { de uma tática ou planejamento } \\
\text { voltado a saúde física e mental } \\
\text { dessas gestantes, que são tão } \\
\text { excepcionais quanto qualquer um } \\
\text { outro grupo observado pela } \\
\text { atenção pública. }\end{array}$ & $\begin{array}{l}\text { As análises apontam que a neoplasia da mama em gestantes tende a } \\
\text { se expandir com o passar dos anos, em conciliação com a gestante } \\
\text { precoce, predisposição genética e praxes no cotidiano da mulher } \\
\text { moderna. O estudo revela que algumas gestantes ao serem } \\
\text { diagnosticadas frustram-se com a assistência da saúde e exigem } \\
\text { informações e uma autonomia sobre práticas que existem para o } \\
\text { diagnóstico precoce do câncer de mama. Também se conclui que, o } \\
\text { real envolvimento do Enfermeiro no pré-natal, desperta nas } \\
\text { gestantes o sentimento de confiança por sentir segurança no } \\
\text { profissional que presta a assistência para a mãe quanto para o } \\
\text { crescimento do feto. Concluiu que, algumas mulheres ao } \\
\text { descobrirem o câncer, ficaram frustradas com o serviço da saúde, } \\
\text { pela falta de orientaçãa, ausência de informações e diagnóstico } \\
\text { precoce. Nesse estudo participaram de uma intervenção educativa } \\
\text { em grupo focal } 12 \text { gestantes com diagnóstico de câncer de mama. } \\
\text { Onde sabemos que esse diagnóstico inicial proporciona sensação de } \\
\text { insegurança, temor, fragilidade, medo, vergonha, entre outros... }\end{array}$ \\
\hline $\begin{array}{l}\text { GUEDES, } \\
\text { Helena; } \\
\text { DIAS, } \\
\text { Margarida }\end{array}$ & $\begin{array}{l}\text { Gravidez após o } \\
\text { Cancro da Mama: } \\
\text { Estado da Arte }\end{array}$ & 2017 & $\begin{array}{l}\text { Efetua-se a precisão de meta- } \\
\text { análise, estudo longitudinais e } \\
\text { caso-controle, dividido em três } \\
\text { fases e prospetivos, alargados e } \\
\text { prolongados no tempo, na } \\
\text { percepção de alcançar uma maior } \\
\text { segurança na formação de técnicas } \\
\text { e linhas de direções para o clinico } \\
\text { e informações apropriadas e } \\
\text { objetivas para as mulheres } \\
\text { enfermas de cancro da mama. }\end{array}$ & $\begin{array}{l}\text { De acordo com a literatura esse câncer de mama em enfermos mais } \\
\text { novos, retratam o câncer mais agressivos do ponto de vista } \\
\text { biológico. A gestação após o câncer de mama é uma das neoplasias } \\
\text { mais comuns nas mulheres em período de reprodução, sendo que } \\
\text { menos de } 10 \% \text { das mulheres ficaram gestantes após o tratamento } \\
\text { para este câncer, na atualidade está comprovado que nas mulheres } \\
\text { mais novas existe uma maior influência de tumores com subtipos } \\
\text { biológicos com mais hostilidade. O artigo afirma, que } 40 \% \text { a } 50 \% \\
\text { das mulheres pré-menopausicas com antecedentes de câncer de } \\
\text { mama almejam ficar gestantes após o tratamento. A fertilidade, } \\
\text { assim como o material genético herdado são parecidos e } \\
\text { consideráveis, dado que a presença de mutações nos genes BRCA } \\
\text { atinge sobretudo pessoas do sexo feminino com menor idade. A } \\
\text { pesquisa mostra que um levantamento feito pela meta-analise foi } \\
\text { com cinco diferentes estudos, que somavam um total de } 187 \text { doentes } \\
\text { que ficaram gestantes entre } 6 \text { a } 24 \text { meses, e } 353 \text { doentes que } \\
\text { engravidaram dois anos após serem diagnosticadas com câncer de } \\
\text { mama, foi destacado que a gestação mais precoce não mostrava } \\
\text { efeito negativo sobre essa suposição global. }\end{array}$ \\
\hline $\begin{array}{l}\text { ASHWOR } \\
\text { TH, } \\
\text { J. Elizabeth }\end{array}$ & $\begin{array}{l}\text { Cancro da mama } \\
\text { associado à } \\
\text { gravidez - efeito } \\
\text { da quimioterapia } \\
\text { no feto }\end{array}$ & 2016 & $\begin{array}{l}\text { A busca feita através dessa } \\
\text { pesquisa tem como objetivo } \\
\text { constatar sobre a garantia da } \\
\text { exposição da mama a } \\
\text { quimioterapia, visando os } \\
\text { possíveis efeitos a curto e longo } \\
\text { prazo sobre o feto. }\end{array}$ & $\begin{array}{l}\text { A literatura aponta que a gestação não é um impedimento para o } \\
\text { tratamento do câncer, sendo que a taxa de complicação materna } \\
\text { relacionada a terapia quimioterápica no } 2^{\circ} \text { e } 3^{\circ} \text { trimestre é baixa e } \\
\text { que a ameaça de anormalidades fetais não é maior do que o da } \\
\text { população em geral. Para a tomada de decisões terapêuticas é } \\
\text { necessário mostrar todas as possibilidades à gestante, as ameaças e } \\
\text { vantagens. A literatura também mostra que gestantes submetidas a } \\
\text { procedimentos quimioterápicos devem fazer acompanhamento com } \\
\text { hemograma e estudo de função hepática e renal. No decorrer do } \\
\text { tratamento com quimioterapia, as gestantes devem ser expostas as } \\
\text { ecografias adicionais frequentes para ser observado o } \\
\text { desenvolvimento fetal, o líquido amniótico e a função placentária. }\end{array}$ \\
\hline
\end{tabular}




\begin{tabular}{|c|c|c|c|c|}
\hline & & & & $\begin{array}{l}\text { A quimioterapia deve ser pausada pela } 37^{\mathrm{a}} \text { semana para deixar que } \\
\text { a medula óssea recupere, sendo que a partir da } 37^{\mathrm{a}} \text { semana há } \\
\text { qualquer momento pode ocorrer o parto. Verificou-se também que } \\
\text { as crianças nascidas de mulheres com câncer de mama são mais } \\
\text { pequenas para a idade gestacional do que as crianças cujas as mães } \\
\text { não têm o diagnóstico de câncer, independentemente de nascerem a } \\
\text { termo }(15,4 \% \text { vs } 11,1 \%) \text { ou antes do termo }(26,7 \% \text { vs } 18,3 \%) \text {. }\end{array}$ \\
\hline $\begin{array}{l}\text { SILVA, } \\
\text { Priscila; } \\
\text { et al }\end{array}$ & $\begin{array}{l}\text { Câncer de mama } \\
\text { na gestação: } \\
\text { Diagnóstico } \\
\text { tratamento }\end{array}$ & 2017 & $\begin{array}{l}\text { Tem como objetivo sanar } \\
\text { mecanismos de diagnóstico do } \\
\text { câncer de mama na gravidez. } \\
\text { Observar os tipos de tratamento } \\
\text { indicados para câncer de mama na } \\
\text { idade gestacional. }\end{array}$ & $\begin{array}{l}\text { Ao analisar o artigo, percebe-se que o diagnóstico do câncer de } \\
\text { mama no período gestacional é o exame clínico mamário e o exame } \\
\text { de imagem, também pode ser feito por anamnese. Tendo em vista, } \\
\text { que tratamento precisa ser feito de forma minuciosa, sempre } \\
\text { observando em função dos potenciais efeitos adversos para não } \\
\text { prejudicar o feto. Procurou-se esclarecer o conhecimento sobre o } \\
\text { diagnóstico que pode ser feito através de exames de imagens como: } \\
\text { ultrassonografia de mama e ressonância magnética das mamas; há } \\
\text { opções terapêuticas como as cirurgias e radioterapia. Nas } \\
\text { quimioterapias é proibido a amamentação. A radioterapia não é } \\
\text { aconselhada, porém em pequenas doses pode ser usada para emitir } \\
\text { raios de luz em algumas regiões maternas sem comprometer o feto. }\end{array}$ \\
\hline $\begin{array}{l}\text { FONTES, } \\
\text { Adriele; } \\
\text { et al }\end{array}$ & $\begin{array}{l}\text { Câncer de mama } \\
\text { na gestação tardia }\end{array}$ & 2017 & $\begin{array}{l}\text { Buscar conhecer indicadores de } \\
\text { grande valia disponíveis na } \\
\text { literatura para sanar dúvidas sobre } \\
\text { a associação entre câncer e } \\
\text { gestação, para poder compreender } \\
\text { a relação entre os mesmos. }\end{array}$ & $\begin{array}{l}\text { Observou-se que as alterações fisiológicas que tornam o diagnóstico } \\
\text { dificultoso nessas pacientes com câncer de mama no período } \\
\text { gestacional são: hipervascularização, hipertrofia e ingurgitamento. } \\
\text { O câncer mamário no decorrer do período fértil da mulher se } \\
\text { apresenta em } 10 \text { a } 20 \% \text {, e de } 3 \text { a } 8 \% \text { das mulheres diagnosticadas } \\
\text { pela doença se tornarem gestantes. Ao ser diagnosticada com câncer } \\
\text { de mama durante o período gestacional, a mulher não pode fazer o } \\
\text { tratamento com quimioterapia logo de imediato. É necessário } \\
\text { aguardar o segundo trimestre da gestação, pois no primeiro } \\
\text { trimestre, a gestação é bastante arriscada e o desenvolvimento do } \\
\text { feto exige proteção, atenção e cuidado. Também é importante frisar } \\
\text { sobre o exame da mamografia que não é indicada no ciclo } \\
\text { gestacional devido as suas alterações fisiológicas, como o aumento } \\
\text { do tamanho da vascularização. }\end{array}$ \\
\hline $\begin{array}{l}\text { VILLAR, } \\
\text { Raquel; } \\
\text { et al }\end{array}$ & $\begin{array}{l}\text { Qualidade de vida } \\
\text { e ansiedade em } \\
\text { mulheres com } \\
\text { câncer de mama } \\
\text { antes e depois do } \\
\text { tratamento }\end{array}$ & 2017 & $\begin{array}{l}\text { O objetivo é demonstrar a } \\
\text { qualidade de vida dessa paciente } \\
\text { na prática clínica e diária, sobre os } \\
\text { dados que influenciam as tomadas } \\
\text { de decisões, intervenções } \\
\text { médicas, } \\
\text { biomédicas. }\end{array}$ & $\begin{array}{l}\text { Foi investigado que após os resultados dos tratamentos, as medidas } \\
\text { alteradas em caráter negativo e consideráveis foram a função física, } \\
\text { funcional, fadiga, imagem corporal, dor, dispneia, e dificuldade } \\
\text { financeira. O tratamento pode ser modificado de acordo com a } \\
\text { qualidade de vida dessa mulher com câncer na gravidez. A pesquisa } \\
\text { indica dados sobre qualidade de vida que persuadiram a tomada de } \\
\text { decisões em } 30,1 \% \text { das cirurgias médicas e em } 63,2 \% \text { dos } \\
\text { tratamentos não biomédicas. }\end{array}$ \\
\hline
\end{tabular}

Fonte: Autores a partir de síntese de artigos selecionados para a composição da pesquisa.

Os artigos selecionados no Quadro 1 tem ligação direta com o Cancer de mama no periodo gestacional, e a partir de uma leitura minuciosa e analítica, foram ressaltados pontos de relevancia que contribuem para o discernimento dos profissionais e fatores que rodeiam a tematica em questão. Portanto, continuamente serão abordados tópicos com análise quantitativa e qualitativa, com o intuito de enfatizar pontos cruciais e de extremo interesse para o estudo em quetão.

\subsection{Câncer de Mama}

De acordo com o Instituto Nacional do Câncer (2021), o CA de mama é a neoplasia com maior incidência entre as mulheres no mundo, equivalente a 2,3 milhões de casos recentes registrados em 2020, o que significa que 24,5\% das mulheres foram recentemente afetadas pelo câncer de mama. Vale ressaltar que é o fator de mortalidade por câncer mais constante na população feminina.

O câncer de mama no território Brasileiro é a categoria de câncer com maior incidência em mulheres, atrás apenas do câncer de pele. Considerando que nas regiões de maior desenvolvimento (sudeste e sul), o percentual é mais expressivo. Entre 2020 e 2022, a estimativa é equivalente a 66.280 casos novos de câncer de mama em mulheres (Instituto Nacional de Câncer 2021).

Segundo Azevedo et al (2017), o câncer de mama é uma doença causada pela multiplicação desordenada de células mamarias, em forma de tumor, sendo um dos mais temidos entre a população feminina, podendo também cometer homens. Os exames recomendados para investigação pode ser o exame clínico das mamas (palpação) e exames de imagens, como 
mamografia, ultrassonografia ou ressonância magnética. Em razão de sua elevada incidência e, sobretudo, pelo impacto psicológico e social que ocasiona, principalmente em decorrência dos medos e tabus que cercam essa doença.

Para Pedrosa et al (2020), câncer é o nome dado a um conjunto de mais de 100 doenças que tem em comum o crescimento desordenado das células, que invadem os tecidos e os órgãos. Embora, existem muitos tipos de cânceres, todos começam devido ao crescimento e multiplicação desordenada das células, formando tumores. O câncer de mama é diagnosticado em 1 em cada 3.000 mulheres grávidas. Esse tipo de neoplasia vem crescendo de forma gradativa, pois, as mulheres estão optando por adiar cada vez mais a gestação. Portanto, é importante destacar que a idade materna avançada está fortemente associada ao risco de desenvolver neoplasias mamárias durante a gestação.

Para Codorniz et al. (2016), o câncer de mama é o tipo de tumor mais comumente detectado durante a gravidez. Equivalente a 40-50\% das mulheres que sobrevivem do CA mamário, almejam engravidar após o termino do tratamento. Essa baixa taxa pode ser explicada por vários fatores, como o declínio da fertilidade relacionado ao tratamento. No tratamento do câncer de mama durante a gravidez, a mastectomia e a cirurgia conservadora são opções quando o diagnóstico é feito no primeiro trimestre da gravidez. No segundo e terceiro trimestres, a quimioterapia neoadjuvante pode ser utilizada para o tratamento, ou seja, os medicamentos de tratamento são administrados antes do tratamento principal.

Cunha e Soares (2017), afirmam que o câncer de mama na gestação é aquele que ocorre durante a gravidez ou até um ano após o parto. É o tipo de câncer que mais acomete as mulheres em todo o mundo; no Brasil perde somente para o câncer do colo de uterino. Trata-se de uma neoplasia maligna, sua incidência estima-se em 1/3.000 a 1/10.000 gestações, tornando o cancro da mama umas das patologias malignas mais frequente durante a gestação.

Essa neoplasia da mama quando diagnosticada em idade mais jovens tendem a ser mais agressivas, assim como o cancro da mama associado a gravidez é geralmente mais agressivo do que nas pacientes não grávidas. O diagnóstico do cancro da mama associado a gravidez é feito de forma semelhante ao diagnóstico desta neoplasia fora da gravidez. O seu diagnóstico tende ser mais tardio devido as alterações fisiológicas, sendo também mais difícil a interpretação dos exames de diagnóstico (Ashworth, 2017).

Outro dado bastante relevante trazido por Silva et al (2017), é que a maioria dos cânceres de mama durante a gestação é a do tipo adenocarcinoma ductal infiltrante, isso se deve pelo diagnóstico tardio limitado pelas alterações fisiológicas da gestação. Com isso, o diagnóstico durante a gravidez pode ser mais difícil devido as alterações fisiológicas mamárias que ocorrem nesta fase como hipertrofia e modularidade, bem como pela incerteza e precisão dos meios diagnósticos que podem ser realizados através da ultrassonografia de mama, ressonância magnética das mamas e punção aspirativa por agulhas finas.

Por outro lado, Fontes et al (2017), diz que o câncer de mama na gestação está se tornando algo frequente em meio a sociedade, devido as mudanças comportamentais das mulheres na atualidade. Em relação ao tratamento do CA no período gravídico, a quimioterapia não é a primeira opção no primeiro momento, pois é necessário a chegada do segundo trimestre da gestação, pois é no primeiro trimestre que o desenvolvimento do feto requer mais atenção e proteção. A radioterapia só pode ser realizada em períodos após o parto, devido à alta concentração de radiação emitida, podendo influenciar na má formação do fetal.

\subsection{Período Gestacional}

Para Alves e Bezerra (2020), durante o período gestacional, o organismo da mulher passa por uma série de alterações e adaptações, medidas por fatores hormonais ou mecânicos. Entre as mudanças hormonais, destacam-se àquelas oriundas da ação da relaxina que aumenta a frouxidão ligamentar e entre as mudanças mecânicas é importante ressaltar aquelas que provocam modificações estruturais na estática e dinâmica do esqueleto, principalmente relacionado ao ângulo lombo-pélvico que aumenta a medida que a gestação avança. $\mathrm{O}$ aumento da curvatura lombar ocorre como mecanismo compensatório ao aumento dos seios, 
do útero gravídico, do peso da mãe e do bebê, além do aumento do líquido amniótico e da circunferência abdominal e tendem a se agravar, uma vez que são progressivas com o avanço da gestação.

Segundo Cestári et al (2017), na gestação ocorre periodos de mudanças fisiológicas, sociais, familiares e psicológicas, podendo ser um período em que a mulher pode desencadear transtornos psiquiátricos, sendo um deles a depressão, acometendo o período gestacional ou o puerpério, desencadeando uma repercusão negativa na qualidade de vida da gestante ou da puerpera.

De acordo com Horlanda (2017), em decorrencia da gravidez e lactação, as mamas sofrem mudanças de grande importância, totamente não conhecidas, mas que se desencadeam através das mudanças hormonais, que formam a hiperplasia vascular e lobular. Com a influência do estrogênio, no primeiro trimestre do periodo gravídico ocorre a ramificação do sistema ductal, em conjunto com o crescimento dos lóbulos, associado com o aumento da vascularização do fluxo sanguíneo em volta da mama.

Para Mayan et al (2019), com as mudanças das mamas na fase gestacional, o crescimento dos lóbulos, ao exame físico durante a palpação pode ser confundido com os nódulos; caso haja presença de nódulos eles podem ser menos notados, pois na gravidez as mamas se tornam mais densas e dolorosas. O câncer de mama associado a gestação pode passar desapercebido pelas alterações fisiológicas próprias da gravidez.

Segundo Silva et al (2018), o câncer mamário durante a gravidez é uma condição rara. A apresentação típica do câncer de mama durante a gravidez é a mesma que em mulheres não grávidas: uma massa palpável e indolor. Recomenda-se que na primeira consulta do pré-natal, seja realizado um exame físico completo da mulher, incluindo a palpação das mamas e axilas. Quando uma mulher grávida é diagnosticada com câncer de mama, é considerado câncer de mama durante a gravidez ou câncer de mama relacionado à gravidez.

De acordo com Monteiro et al (2017), a gestação em si é um período bem sensível para a mulher e seus familiares, e a gestação acompanhado do CA de mama se torna um momento ainda mais sensível e desesperador. A ocorrência do câncer no período gestacional é relacionada a idéia de uma doença com comportamento muito agressivo, onde as grávidas sentem medo, insegurança ao serem diagnosticadas, pois a maior preocupação é o impacto da doença, com incerteza sobre sua vida e do feto e isso, lhes causa traumas e desespero.

Para Lima, Steger e Pontes (2019), a gestante com CA necessita de um pré-natal mais específico, controlado, com rotina de pré-natal direcionada, garantindo umdesenvolvimento fetal adequado. A equipe deve ser especializada e deve ser composta tanto pelo obstetra quanto 'por oncologista, mastologista, nutricionista, psicólogo, assistente social, ou seja, uma equipe multiprofissional. As gestantes que descobrem o CA durante a gravidez, tem uma evolução da gravidez e do câncer muito favorável. Há 20 anos, a conduta recomendada era o aborto terapêutico, as gestantes optavam ou eram convencidas a realizar o aborto, por conta do tratamento de câncer, e estas tinham uma evolução pior do câncer do que aquelas que conseguiam manter a gravidez.

\subsection{Atuação do profissional enfermeiro na assistência a gestantes com câncer de mama}

Em acordo com Lélis et al (2019), o papel do enfermeiro na condução de situações ligadas ao câncer gestacional é essencial para a paciente. Onde a assistência abrange o diagnóstico, o tratamento e a reabilitação da gestante, devendo focar no esclarecimento sobre a doença e suas opções de tratamento, promovendo informações acerca do autocuidado, oferecendo apoio emocional, proporcionando alívio à dor, incentivando e encorajando a paciente a enfrentar a doença e suas possíveis consequências.

Para Guedes; Figueiredo (2017), grande parte das neoplasias de mama em doentes jovens corresponde a cancros mais agressivos do ponto de vista biológico. Pois o período gestacional proporciona grandes oportunidades para o diagnóstico de câncer, devido à atenção mais minuciosa dada à mulher no período do pré-natal. O profissional de saúde, em especial o 
enfermeiro, deve ter a atenção especial com as gestantes, estando atento para qualquer alteração detectada no período gestacional, contribuindo, assim no controle da doença, por meio de ações de promoção da saúde, prevenção e detecção dos agravos.

$\mathrm{O}$ tratamento de câncer no período gestacional deve ser feito com cautela, avaliando cada caso, com intuito de garantir uma maior sobrevida aos seres envolvidos. Com isso, observa-se a importância de se estudar a doença e seus impactos na gestação, no sentido de sensibilizar os profissionais da área da saúde à grande importância do papel desempenhado pela equipe de enfermagem (Guedes; Figuereido, 2017).

A importância desse profissional na assistência à gestantes, ao que concerne o seu papel de educador em saúde, consiste em oportunizar o ensejo do pré-natal para orientá-las quanto a importância do exame clínico e autoexame das mamas e da realização da colpocitologia, denotando uma melhor apropriação do conhecimento científico e comprometimento ético e profissional junto à população que o assiste, no intuito de amenizar os riscos à saúde materna e otimizar a sobrevida do feto (Rodrigues et al, 2016).

É comprovado por Villar et al (2017) que a qualidade de vida e a ansiedade das pacientes com câncer de mama e as mudanças sofridas após o tratamento são um grande tabu. A maior ansiedade está relacionada às características: sedentarismo no trabalho, ansiolíticos, edema mamário, estágios avançados de diagnóstico, imagem corporal, comportamento sexual e vida materna. Pode-se dizer que esses profissionais precisam estar preparados para orientar e confortar a gestante e seus familiares, pois os mesmos sentirão medo, sentimento de perda, dor e sofrimento. Portanto, acredita-se que o cuidado de enfermagem é de grande importância no acompanhamento e intervenção para as pacientes, sendo as dificuldades do tratamento, a dor e o medo os principais efeitos do tratamento do câncer de mama na gestante devido à dificuldade de aceitação da doença pelas pacientes.

Silva et al (2019), destaca que é caráter do enfermeiro a oferta de ações de atenção integral e multidisciplinar às pacientes que passam por esse período tão delicado do CA na gestação, visando um olhar de qualidade e humanizado no pré-natal, com intuito de ofertar menos desconforto no tratamento dessas gestantes, ressalta-se que o apoio psicológico é primordial a essa situação.

Rodrigues et al (2016), ressaltam que os profissionais se encontram pouco preparados para enfrentar tal situação, o que se torna visível ao certifica-se que é alto o índice das gestantes não diagnosticadas no período gestacional, podendo-se constatar a necessidade da capacitação contínua para todos os profissionais envolvidos com a saúde em gestantes.

Cabe destacar os principais diagnósticos de Enfermagem a serem contemplados, conforme o manejo do Enfermeiro com a assistência de enfermagem à gestante com câncer mamário, que foram destacados no Quadro 2, e são:

Quadro 2: Sistematização da Assistência de Enfermagem no pré-natal, diagnosticada com CA de mama no período gravídico.

\begin{tabular}{|c|c|c|}
\hline Definição & Domínio & Diagnósticos \\
\hline $\begin{array}{l}\text { Nanda 2021-2023 } \\
\begin{array}{l}\text { Domínio 07; Classe } \quad 02 ; \text { Código do } \\
\text { diagnostico } 00063\end{array}\end{array}$ & Relação de função & $\begin{array}{l}\text { Processo familiar disfuncionais relacionado a } \\
\text { habilidade insuficiente para lidar com problemas } \\
\text { evidenciado pela ansiedade. }\end{array}$ \\
\hline $\begin{array}{l}\text { Nanda 2021-2023 } \\
\begin{array}{l}\text { Domínio 09; Classe } 02 ; \text { Código do } \\
\text { diagnostico } 00146\end{array}\end{array}$ & Enfretamento & $\begin{array}{l}\text { Ansiedade relacionado ao conhecimento da CA de } \\
\text { mama evidenciado a preocupação. }\end{array}$ \\
\hline $\begin{array}{l}\text { Nanda 2021-2023 } \\
\begin{array}{l}\text { Domínio 09; Classe } \quad 02 ; \text { Código do } \\
\text { diagnostico } 00148\end{array}\end{array}$ & Enfrentamento & $\begin{array}{l}\text { Medo relacionado a cenário pouco conhecido } \\
\text { evidenciado a diminuição da autoconfiança. }\end{array}$ \\
\hline $\begin{array}{l}\text { Nanda 2021-2023 } \\
\begin{array}{l}\text { Domínio } 11 ; \quad \text { Classe } \quad 02 ; \text { Código do } \\
\text { diagnostico } 00047\end{array}\end{array}$ & Segurança & $\begin{array}{l}\text { Risco de integridade da pele prejudicada evidenciado } \\
\text { a alteração hormonal. }\end{array}$ \\
\hline $\begin{array}{l}\text { Nanda 2021-2023 } \\
\begin{array}{l}\text { Domínio } 12 ; \quad \text { Classe } \quad 01 ; \text { Código do } \\
\text { diagnostico } 00214\end{array}\end{array}$ & Conforto & $\begin{array}{l}\text { Conforto prejudicado relacionado a relacionado a } \\
\text { efeitos das alterações gravídicas e ao CA de mama } \\
\text { evidenciado a náusea, vômitos, ansiedade e medo. }\end{array}$ \\
\hline $\begin{array}{l}\text { Nanda 2021-2023 } \\
\text { Domínio 08; Classe 03; Código do } \\
\text { diagnostico } 00209\end{array}$ & Sexualidade & $\begin{array}{l}\text { Risco de díade materno-fetal perturbada evidenciado a } \\
\text { complicações na gravidez. }\end{array}$ \\
\hline
\end{tabular}

Fonte: Autores a partir do NANDA 2021-2023. 


\section{Considerações Finais}

Por meio do estudo, pode ser observado a frequência e relevância da neoplasia mamária associado ao período gestacional, extremamente relevante para a saúde da mulher no período gravídico. O período gestacional é considerado um estágio muito esperado e desejado pela mulher, onde há um mix de sentimentos, mudanças hormonais e mudanças fisiológicas, e esse período tão delicado associado a uma descoberta de câncer mamário torna tudo duvidoso para essas gestantes, pois o medo e a incerteza da saúde do seu feto e da sua saúde, pode desencadear vários agravos.

Na revisão dos trabalhos presentes na literatura, ficou em evidência que não há uma preparação adequada da assistência do profissional Enfermeiro em gestantes com câncer de mama, pois ainda não é um assunto muito abordado, quando se trata de saúde mãe-feto. Embora seja uma temática pouco explorada, é um assunto de grande relevância para a saúde pública e comunidade.

Contudo, a Enfermagem está de frente ao cuidado diretamente ligado à mulher em todos os níveis de atenção, motivo pelo qual esses profissionais precisam estar qualificados e atentos em todos os níveis de atenção à saúde. Com isso se comprova a relevância do papel do profissional Enfermeiro na assistência, onde não está só envolvido a mulher e o feto, mas também os familiares que acabam ficando vulneráveis. Diante do exposto, assim o Enfermeiro realiza o atendimento comunitário familiar, ofertando apoio emocional, com o intuito do desenvolvimento da gestação de forma saudável com participação familiar, durante o período de enfrentamento contra o câncer mamário. Outra vertente de grande relevância seria a investigações da qualidade de vida dos recém-nascidos, após o tratamento realizado nas puérperas acometidas pelo câncer de mama.

\section{Referências}

Alves, T. V., \& Bezerra, M. M. M. (2020). Principais alterações fisiológicas e psicológicas durante o Período Gestacional. Revista Multidisciplinar e de Psicologia. 14(49), 114-126- ISSN 1981-1179. https://idonline.emnuvens.com.br/id/article/view/2324.

Ashworth, J. E. O. L. (2016). Cancro da mama associado à gravidez- efeito da quimioterapia no feto. https://repositorioaberto.up.pt/bitstream/10216/90024/2/168705.pdf.

Azevedo, D. B., Moreira, J. C., Gouveia, P. A., Tobias, G. C., \& Neto, O. L. M. (2017). Perfil das mulheres com câncer de mama. Revista de Enfermagem UFPE online., Recife, junho de 2017. https://periodicos.ufpe.br/revistas/revistaenfermagem/article/view/23386.

Braga, F. L. B., Sousa, D. M. N., Mendes, M. R., Vasconcelos, C. T. M. \& Oriá, M. O. B. (2018). Orientações de enfermagem para enfrentamento do câncer de mamana gravidez. https://docplayer.com.br/70296586-Orientacoes-de-enfermagem-para-enfrentamento-do-cancer-de-mama-na-gravidez.html.

Cestári, C. E., Souza, T. H. C., Angelo, P. F. C., \& Silva, A. S. (2018). Análise das principais alterações posturais e sintomatologias decorrentes do período gestacional. Revista ciência e estudos acadêmicos de medicina, junho de 2018. https://periodicos.unemat.br/index.php/revistamedicina/article/view/1973/0.

Fontes, A. M., Lima, J. V. S., Santana, S. M. S., Lima, T. A., \& Menezes, M. O. (2017). Câncer de mama na gestação tardia. https://eventos.set.edu.br/cie/article/viewFile/5928/2210.

Cipriano,P. Oliveira, C. (2016). Revisão Gestação e câncer de mama: proposta de guia de orientações Pregnancy and breast cancer: proposal of recomendações. https://portalatlanticaeditora.com.br/index.php/fisioterapiabrasil/article/view/202/1422.

Conordiz, A., Mineiro, S., Esteves, T. Costa, A. \& Fernandes, F. (2016). Gravidez após neoplasia da mama: relato de caso. https://www.researchgate.net/publication/310833888_Gravidez_apos_neoplasia_da_mama_relato_de_caso.

Cunha, F. A. A., \& Soares, S. C. L. V. A. (2017). O desafio do cancro da mama durante a gravidez: Do diagnóstico ao tratamento. https://repositorioaberto.up.pt/bitstream/10216/109164/2/233172.pdf.

Fontes, A. M., Lima, J. V. S., Santana, S. M. S., Lima, T. A. \& Menezes, T. O. (2017). Câncer de mama na gestação tardia. https://eventos.set.edu.br/cie/article/view/5928.

Guedes, H., \& Dias, M. F. (2017). Gravidez após o Cancro da Mama: Estado da Arte. Revista Científica da Ordem dos Médicos, Coimbra Portuga, novembro 2017. https://doi.org/10.20344/amp.8885.

Horlanda, A. A. R. (2017). Alterações fisiológicas mamárias durante a gravidez e lactação: aspectos ultrassonográficos e dopplerfluxométricos. https://1library.org/document/zw347o1y-alteracoes-fisiologicas-mamarias-gravidez-lactacao-aspectos-ultrassonograficos-dopplerfluxometricos.html.

Instituto Nacional de Câncer José Alencar Gomes da Silva. (2020). Estimativa 2020: incidência do Câncer no Brasil. Rio de Janeiro: INCA, 2019. https://www.inca.gov.br/sites/ufu.sti.inca.local/files/media/document/estimativa-2020-incidencia-de-cancer-no-brasil.pdf.

Lélis, B. D. B., Dusso, M. I. S., Souza, F. L. P., \& Bernardes, N. B. (2019). Tratamento do Câncer de Colo do Útero em Gestantes. Revista Multidisciplinar e de Psicologia .13(45), 433-438. https://idonline.emnuvens.com.br/id/article/view/1703/2528. 
Research, Society and Development, v. 10, n. 16, e361101624127, 2021

(CC BY 4.0) | ISSN 2525-3409 | DOI: http://dx.doi.org/10.33448/rsd-v10i16.24127

Lima, V. C. A. Sterger, J. \& Pontes, S. R. (2019). Enfrentamento da mulher com diagnóstico de câncer no período gestacional. Revista da Faculdade União Goyazes, Trindade (GO), 13(2). 2019. http://fug.edu.br/revistas/index.php/VitaetSanitas/article/view/185/160.

Mayan, S. M. G., Barreto, F. L., Paz, S. T., Oliveira, M. T. C. S., \& Fraga, B. G. G. (2019). Câncer gestacional - importância do conhecimento e aprimoramento da equipe de enfermagem. http://www.webfipa.net/facfipa/ner/sumarios/cuidarte/2019v2/165.pdf.

Monteiro, D. L. M., Nunes, C. L., Rodrigues, N. C. P., Antunes, C. A., Almeida, E. M., Barmpas, D. B. S., Magalhães, A. L. C., \& Trajano, A. J. B. (2019). Fatores associados ao câncer de mama gestacional: estudo caso-controle. https://doi.org/10.1590/1413-81232018245.18392017.

NANDA. International Nursing Diagnoses: Definitions \& Classification, 2021-2023.

Pedrosa, A. B., Oliveira, M. G. G., Coelho, V. A. T., Nascimento, E. S., \& Bigatello, C. S. (2018). Métodos terapêuticos indicados no tratamento do câncer de mama gestacional. Revista Multidisciplinar do Nordeste Mineiro, 2020/02.

https://revistas.unipacto.com.br/storage/publicacoes/2020/500_metodos_terapeuticos_indicados_no_tratamento_do_cancer_de_mama_gestaci.pdf.

Prado, N., Loiola, P. Guimarães, T. Ohara, E. C. C., \& Oliveira, L. D. R., (2020). Gestante com diagnóstico de câncer de mama: prevenção, diagnóstico e assistência. https://www.brazilianjournals.com/index.php/BJHR/article/view/6875/6062.

Rodrigues, C. M. O., Maximino, D. A. F. M., Souto. C. G. V. \& Virgínio, N. A. (2016). Repercussão do tratamento das neoplasias durante a gestação. Revista Ciência Saúde Nova Esperança, Abril 2016; http://www.facene.com.br/wp-content/uploads/2010/11/7.-REPERCUSS\%C3\%83O-DO-TRATAMENTO-DASNEOPLASIAS_PRONTO.pdf..

Silva, K. M., Rockenbach, B. F., Moura, J. E. \& Souza, A. B. A. (2018). Câncer de mama na gestação: abordagem diagnóstica e terapêutica. https://pesquisa.bvsalud.org/portal/resource/pt/biblio-987625.

Silva, L. P., Gomes, R. S., \& Silva, T. K. E. (2019). Assistência de enfermagem no tratamento e prevenção de câncer de mama gestacional: revisão integrativa. http://65.108.49.104/bitstream/123456789/104/1/TCC\%20-\%20LEIDIANNY\%2C\%20ROBERTA\%2C\%20THAYS.doc-compactado.pdf.

Silva, P. T. A., Loiola, P. H., \& Santoa, V. R. (2017). Câncer de mama na gestação:diagnóstico e tratamento. https://docplayer.com.br/95922259-Titulopesquisa-de-estreptococos-do-grupo-b-em-gestantes-categoria-concluido-area-ciencias-biologicas-e-saude-subarea-biomedicina.html.

Soares, S. V. Picolli, I. R. A., \& Casagrande, J. (2018). Pesquisa bibliográfica, pesquisa bibliométrica, artigo de revisão e ensaio teórico em administração e contabilidade. http://www.spell.org.br/documentos/ver/50255/pesquisa-bibliografica--pesquisa-bibliometrica.

Vilar, R. R., Fernández, S. P., Garea, C. C., Pillado, M. T. S., Barreiro, V. B., \& Martín, C. G. (2017). Qualidade de vida e ansiedade em mulheres com câncer de mama antes e depois do tratamento. Revista Latino Americano de Enfermagem, 2017. https://www.scielo.br/j/rlae/a/b4kQpywJX5jPstMFnGypfGN/?lang=pt\&format=pdf. 